\title{
Difficulties in a Kind of Averaging Procedure for Constructing Gauge-invariant Operators out of Gauge-variant Ones
}

\author{
Wei-Min Sun ${ }^{a}$, Xiang-Song Chen ${ }^{a, b}$ and Fan Wang ${ }^{a}$ \\ ${ }^{a}$ Department of Physics and Center for Theoretical Physics, Nanjing University, Nanjing 210093, China \\ ${ }^{b}$ Institut für Theoretische Physik, Universität Tübingen, Auf der Morgenstelle 14, D-72076 Tübingen, Germany
}

\begin{abstract}
We prove that a kind of averaging procedure for constructing gauge-invariant operators(or functionals) out of gauge-variant ones is erroneous and inapplicable for a large class of operators(or functionals).
\end{abstract}

PACS: $11.15 .-\mathrm{q}, 12.20-\mathrm{m}$

Gauge invariance is a basic requirement in gauge theories. Only gauge-invariant operators correspond to physical observables. Then how to construct gauge-invariant operators(or functionals) out of gauge-variant ones is of fundamental importance since the advent of these theories. In the literature this problem has been intensively studied. For example in [1] a kind of dressing is used to construct gauge-invariant constituent quark and gluon fields out of gauge-variant bare ones. In the literature there also exist a kind of recipe of formally averaging out any gauge dependence to produce a gauge invariant functional [2]. In this paper we will show that the latter method is erroneous and inapplicable for a large class of operators(or functionals). Here we restrict ourself in quantum electrodynamics.

First let us describe this averaging procedure. For an arbitrary functional $O[\phi]$, gauge invariant or not, we can always define a functional $G_{O}[\phi]=\int D \omega O\left[\phi^{\omega}\right]$ where $\phi$ denotes gauge or matter fields, $\phi^{\omega}$ denotes the result of $\phi$ after a gauge transformation $\omega$ and $\int D \omega$ stands for functional integration over the gauge group(here we choose the normalization so that $\left.\int D \omega=1\right)$. Using the property $\int D \omega f(\omega)=\int D \omega f\left(\omega_{0} \omega\right)$ we can easily prove that $G_{O}[\phi]$ is gauge-invariant:

$$
\begin{aligned}
G_{O}\left[\phi^{\omega_{0}}\right] & =\int D \omega O\left[\phi^{\omega_{0} \omega}\right] \\
& =\int D \omega O\left[\phi^{\omega}\right] \\
& =G_{O}[\phi]
\end{aligned}
$$

This procedure formally gives a gauge-invariant functional. One nontrivial example is provided by the FaddeevPopov trick [3] in quantizing a gauge field theory using functional integral methods. The Faddeev-Popov determinant $\Delta_{F}[A]$ defined by

$$
\Delta_{F}[A] \int D \omega \delta\left(F\left[A^{\omega}\right]\right)=1
$$

is gauge-invariant where $F[A]$ is a suitable gauge-fixing functional.

In the following we will show that for a class of functionals this kind of averaging procedure is erroneous and inapplicable.

First let us take $O[\phi]$ to be $A^{\mu}(x)$. In this case we will show that the averaging procedure is inapplicable. Now $O\left[\phi^{\omega}\right]=A^{\mu}(x)+\partial^{\mu} \theta(x)$ and formally integrating over $\omega$ yields

$$
G_{O}[\phi]=A^{\mu}(x)+\int D \omega \partial^{\mu} \theta(x)
$$

From this we see that the averaging procedure gives a gauge-variant operator! Then where do we go wrong? The reason is the following: one can show that the functional integration $\int D \omega \partial^{\mu} \theta(x)$ cannot be consistently defined [4]. Here we present the essential arguments. In any viable definition of functional integration over the gauge group the two properties $\int D \omega f(\omega)=\int D \omega f\left(\omega^{-1}\right)$ and $\int D \omega f\left(\omega_{0} \omega\right)=\int D \omega f(\omega)$ must hold. Noting that $\partial^{\mu} \theta(x)=$ $-i \omega^{-1}(x) \partial^{\mu} \omega(x)$, where $\omega(x)=e^{i \theta(x)}$, we have

$$
\int D \omega \partial^{\mu} \theta(x) \sim \int D \omega \omega^{-1}(x) \partial^{\mu} \omega(x)
$$

Now we can show that the above mentioned two properties cannot simultaneously be satisfied when the integrand is $\omega^{-1}(x) \partial^{\mu} \omega(x)$. If we require the first property to hold this integration should be zero: 


$$
\begin{aligned}
\int D \omega \omega^{-1}(x) \partial^{\mu} \omega(x) & =\int D \omega \omega(x) \partial^{\mu} \omega^{-1}(x) \\
& =-\int D \omega \omega^{-1}(x) \partial^{\mu} \omega(x)
\end{aligned}
$$

If we require the second property to hold this integration should be infinity:

$$
\begin{aligned}
\int D \omega \omega^{-1}(x) \partial^{\mu} \omega(x) & =\int D \omega\left(\omega_{0}(x) \omega(x)\right)^{-1} \partial^{\mu}\left(\omega_{0}(x) \omega(x)\right) \\
& =\int D \omega\left(\omega_{0}^{-1}(x) \partial^{\mu} \omega_{0}(x)+\omega^{-1}(x) \partial^{\mu} \omega(x)\right) \\
& =\omega_{0}^{-1}(x) \partial^{\mu} \omega_{0}(x)+\int D \omega \omega^{-1}(x) \partial^{\mu} \omega(x)
\end{aligned}
$$

So from the above analysis we see that the functional integration $\int D \omega \partial^{\mu} \theta(x)$ cannot be consistently defined. Now the situation is clear. The operator $\int D \omega O\left[\phi^{\omega}\right]$ does not exist so of course we cannot talk about its gauge invariance. Therefore in this case the averaging procedure is erroneous and inapplicable.

In the following we will investigate a general functional of $A^{\mu}(x)$ of the form:

$$
K[A ; x]=K^{(0)}(x)+\int d y K_{\mu}^{(1)}(y ; x) A^{\mu}(y)+\frac{1}{2 !} \iint d y d z K_{\mu \nu}^{(2)}(y, z ; x) A^{\mu}(y) A^{\nu}(z)+\ldots
$$

where $K^{(0)}(x), K_{\mu}^{(1)}(y ; x), K_{\mu \nu}^{(2)}(y, z ; x)$ etc. are suitable smooth functions or distributions in their arguments. We also assume that $K^{(1)}, K^{(2)}$ etc. have compact supports with respect to the variables $y, z$ etc. Functionals expressible in this form should be called analytic functionals.

Now we will derive a criteria for the gauge invariance of a general analytic functional. For this purpose we do an infinitesimal gauge transformation $\left(A^{\mu}(y)\right)^{\theta}=A^{\mu}(y)+\partial^{\mu} \theta(y)$ and take $K[A ; x]=K\left[A^{\theta} ; x\right]$. Expanding both sides in terms of $A^{\mu}(y)$ and comparing the zeroth order, first order $\cdots$ terms gives(here we only keep $\theta(y)$ up to first order because it is infinitesimal):

$$
\begin{gathered}
K^{(0)}(x)=K^{(0)}(x)+\int d y K_{\mu}^{(1)}(y ; x) \partial^{\mu} \theta(y) \\
\int d y K_{\mu}^{(1)}(y ; x) A^{\mu}(y)=\int d y K_{\mu}^{(1)}(y ; x) A^{\mu}(y)+\frac{1}{2 !} \iint d y d z K_{\mu \nu}^{(2)}(y, z ; x)\left(A^{\mu}(y) \partial^{\nu} \theta(z)+\partial^{\mu} \theta(y) A^{\nu}(z)\right) \\
\ldots .
\end{gathered}
$$

Using eq(8) and integrating by parts gives(this is always possible because $K_{\mu}^{(1)}(y ; x)$ has compact support with respect to $y$ )

$$
\partial_{(y)}^{\mu} K_{\mu}^{(1)}(y ; x)=0
$$

Similarly from eq(9) and using the symmetric property $K_{\mu \nu}^{(2)}(y, z ; x)=K_{\nu \mu}^{(2)}(z, y ; x)$ we get $\int d y K_{\mu \nu}^{(2)}(y, z ; x) \partial^{\mu} \theta(y)=0$. Using this equation and integrating by parts(for the same reason as above) gives

$$
\partial_{(y)}^{\mu} K_{\mu \nu}^{(2)}(y, z ; x)=0
$$

(Note that from the symmetric property of $K^{(2)}$ this equation automatically implies $\left.\partial_{(z)}^{\nu} K_{\mu \nu}^{(2)}(y, z ; x)=0\right)$. From this kind of reasoning we can obtain the criteria for the gauge invariance of an analytic functional $K[A ; x]$

$$
\begin{aligned}
\partial_{(y)}^{\mu} K_{\mu}^{(1)}(y ; x) & =0 \\
\partial_{(y)}^{\mu} K_{\mu \nu}^{(2)}(y, z ; x) & =0 \quad \& \quad \partial_{(z)}^{\nu} K_{\mu \nu}^{(2)}(y, z ; x)=0 \\
\ldots \ldots &
\end{aligned}
$$

From this criteria we can see that an analytic functional is gauge-invariant if and only if each term in its Taylor expansion is gauge-invariant separately. 
Now let us consider a polynomial-type analytic functional(an analytic functional with only finite terms in its Taylor expansion) and assume it is gauge-variant. It is possible that some terms of it is gauge-invariant. If this is the case we can drop these gauge-invariant pieces because they do not affect the averaging procedure. Then we get a gauge-variant polynomoial-type analytic functional with its highest order term in $A$ being gauge-variant. Now it is easy to see that the problem encountered in the averaging procedure for $A^{\mu}(x)$ (the undefinability of the relevant functional integral) must also exist in this case. Because if it does not formally integrating with respect to $\omega$ would yield a polynomial-type analytic functional with the same highest order term as the original one, which must then be gauge-variant. That is to say, in this case the averaging procedure must be erroneous and inapplicable.

From the above analysis we can draw a conclusion: the averaging procedure is inapplicable for any gauge-variant local operator made out of the $A$ field and/or its derivatives. This is because any such local operator can be expressed as some polynomial-type analytic functional in $A$.(here is an examples: $(\partial \cdot A(x))^{2}=\iint d y d z \partial_{\mu}^{(x)} \delta(y-x) \partial_{\nu}^{(x)} \delta(z-$ $\left.x) A^{\mu}(y) A^{\nu}(z)\right)$.

Now we have proved that the averaging procedure is inapplicable for any gauge-variant polynomial-type analytic functional. In other words for the averaging procedure to be physically meaningful we must pay attention to analytic functional with infinite terms in its Taylor expansion [2] or non-analytic functionals(for example the case of FaddeevPopov trick where a distribution in $A$ is involved).

In summary we have proved that the averaging procedure for constructing gauge-invariant functionals(or operators) out of gauge-variant ones must be inapplicable for a class of functionals(gauge-variant polynomial-type analytic functional in gauge fields)and especially for any gauge-variant local operator made out of gauge field and/or its derivatives. This work is supported in part by the NSF(19675018), SED and SSTD of China, and in part by the DAAD.

[1] M.Lavelle and D.McMullan, Phys. Rept. 279 (1997) 1-65.

[2] I.I.Kogan and A.Kovner, Phys. Rev. D51 (1995) 1948.

[3] See, for instance L.D.Faddeev and A.A.Slavnov Gauge Fields, Introduction to Quantum Theory, second edition (AddisonWesley, 1991)

[4] W.M.Sun, X.S.Chen and F.Wang, hep-th0003279 